\begin{tabular}{|c|l|}
\hline Title & Picosecond gain spectroscopy of a laser dye during mode locked laser action \\
\hline Author(s) & Y amashita, Mikio; Sato, Takuzo \\
\hline Citation & $\begin{array}{l}\text { A pplied Optics, 21(12), 2092-2095 } \\
\text { https://doi.org/10.1364/A0.21.002092 }\end{array}$ \\
\hline Issue Date & 1982-06-15 \\
\hline Doc URL & http://hdl.handle.net/2115/45315 \\
\hline Rights & ○ 1982 Optical Society of A merica \\
\hline Type & article \\
\hline File Information & A 021-12_2092-2095.pdf \\
\hline
\end{tabular}

Instructions for use 


\section{Picosecond gain spectroscopy of a laser dye during mode-locked laser action}

\section{Mikio Yamashita and Takuzo Sato}

Electrotechnical Laboratory, Radio- \& Optoelectronics Division, Laser Research Section, 1-1-4 Umezono, Sakura-Mura, Niihari-gun, Ibaraki-ken 305, Japan.

Received 16 November 1981.

0003-6935/82/122092-04\$01.00/0.

(C) 1982 Optical Society of America.

Recently synchronously or synchronous-passive hybrid mode-locked (HML) cw dye lasers were used as reliable sources of picosecond and subpicosecond pulses tunable in the visible and near-IR regions. ${ }^{1-5}$ In addition, continuous trains of UV picosecond pulses have been produced by intracavity second harmonic generation (SHG) in a synchronous modelocked (SML) cw dye laser ${ }^{6}$ and employed for studying fast photochemical reactions of molecules. ${ }^{7}$ In all the cases it was reported that the pulse duration and shape are critically dependent on the dye laser cavity length. ${ }^{1-6,8-10}$ That is, generation of stable tunable ultrashort pulses is closely related to the time of arrival of the dye pulse at the dye jet after the round trip in the cavity with respect to the time evolution of the gain induced by the pump pulse. The subject of this Letter is to disclose experimentally the relationship between gain behavior varying on a picosecond time scale and pulse formation for cavity length detuning using continuous pump-probe gain spectroscopy. ${ }^{11}$

The experimental arrangement for simultaneous measurements of the gain evolution and autocorrelation trace of the pulse vs cavity length detuning is shown in Fig. 1. A mode-locked Ar-ion laser (Spectra-Physics 171-19UV) with the average power of $1.6 \mathrm{~W}$ at $514.5 \mathrm{~nm}(\sim 150$-psec pulse duration) is used as the excitation source of a separation-type synchronous-passive HML $\mathrm{cw}$ dye laser providing probe pulses. The gain sample to be measured is the lasing dye itself ( $1 \times 10^{-3}-\mathrm{M}$ rhodamine $6 \mathrm{G}$ in ethylene glycol) of the HML dye laser. The cavity of the HML laser consists of the two concave mirrors for focusing the excitation pulse on the laser dye and collimating the dye laser beam, two other concave mirrors for focusing the laser beam on the saturable dye $(8 \times$ $10^{-6}-\mathrm{M}$ DODCI in ethylene glycol), and an output mirror $(\sim 10 \%$ transmission at $600 \mathrm{~nm})$. The wavelength of the laser 


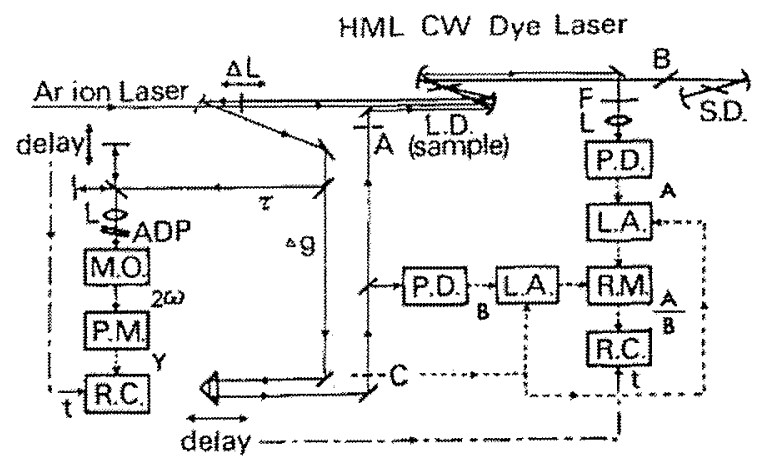

Fig. 1. Schematics of experimental arrangement for the simultaneous measurement of the gain evolution and autocorrelation trace during separation-type HML action.

is changed by a Brewster-angle birefringent filter $0.3-\mathrm{mm}$ quartz crystal) in the cavity. From this laser system a pulse duration of $0.4 \mathrm{psec}$ was obtained at maximum, which was determined from the autocorrelation method using SHG of the first kind from $0.5-\mathrm{mm}$ thick ADP crystal. ${ }^{5}$ At the present operational condition under the high pumping power of more than twice the threshold power, pulses of $\sim 0.8$-psec duration and average power of $\sim 50 \mathrm{~mW}$ are produced at $572 \mathrm{~nm}$. The dye laser output beam is split into two beams (3:2): one main beam, employed for probing the gain evolution, is optically delayed by moving the reflection prism after being chopped; the other beam is used to measure the pulse duration by the autocorrelation method. A portion of the probe beam focused onto the lasing dye (sample) is detected by a photodiode to remove the effect of the variation in dye laser power. The electric output processed by a lock-in amplifier (PAR 126) is fed to the denominator input of a ratiometer (PAR 188) for normalizing the gain signal. The main portion of the probe beam is focused onto the lasing dye jet by one of the concave mirrors. The amplified beam after being collimated by the other concave mirror passes through the color separation glass filter being used to remove the green pump beam and is then focused onto a photodiode through a lens. The electric signal after passing through another lock-in amplifier (PAR 124A) is normalized by being connected to the numerator input of the ratiometer followed by a chart recorder. The induced gain is recorded as a function of the optical delay of the probe beam.

Figure 2 shows a series of the time evolutions of the gain and the pulse autocorrelation traces obtained at different settings of the cavity length during ML laser action. The number to the right of each datum is the reading (in micrometers) of the cavity length adjustment $\Delta L$ with larger numbers corresponding to increasing cavity length. The reference point of $\Delta L=0$ is taken at the point of the cavity length where the intensity of the SH wave in the autocorrelation measurement is maximum. The indicated pulse duration is determined by assuming a single-sided exponential pulse shape. ${ }^{1-6}$ From the observed gain evolution it is found that a sharp dip in the vicinity of the gain peak occurs. This gain drop is caused by a loss in population inversion due to stimulated emission occurring when the intracavity dye pulse strikes the dye jet. Therefore, the arrival time of the dye pulse, which is shown by dashed arrows in Fig. 2, can be distinctly defined from the gain evolution.

As is evident from the uppermost gain evolution and autocorrelation trace of the left side in Fig. 2, when the cavity

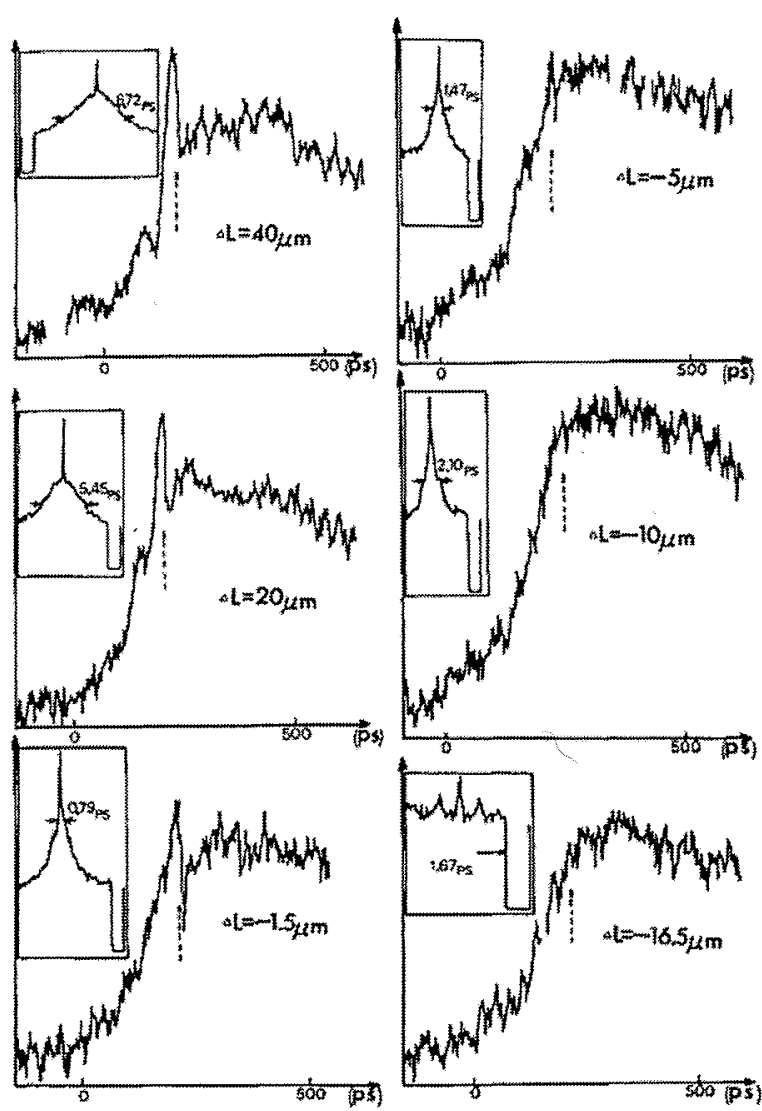

Fig. 2. Series of simultaneously measured gain evolutions and autocorrelation traces during separation-type HML action at different settings of the cavity length $\Delta L$. Dashed arrows represent the arrival time of the dye pulse at the dye jet.

length is too long a sharp cone occurs in the vicinity of the gain peak while, as for the autocorrelation trace, a broad pedestal betraying the presence of substructure in the pulse occurs. The gain evolution is similar to the result obtained from the fluorescence signal from the dye jet by an optical light-gate technique ${ }^{8}$ except that the gain cone is much sharper. The difference is presumably due to the present technique being better in time resolution than the light-gate technique. As the cavity length is shortened the cone is sharpened, while the pedestal becomes less broad indicating a $\sim 5.5$-psec pulse duration of still with a structure. For cavities slightly shorter $(\Delta L=-1.5 \mu \mathrm{m})$ than for maximum SHG, the height of the cone is relatively lowered as high as the following recoveredgain level, while the substructure in the pulse is suppressed and the shortest pulse of 0.79 -psec duration is produced. Further shortening of the cavity (from $\Delta L=-5$ to $-10 \mu \mathrm{m}$ ) continues to lower the cone height of the gain and broaden the pulse duration gradually to 2.1 psec. For the shortest cavities of $\Delta L=-16.5 \mu \mathrm{m}$, the cone of the gain remains obscure in the gain rising process, while the autocorrelation trace does not show the contrast ratio of 3:1 and satellite pulses occur. As for a series of autocorrelation traces this behavior agrees well with already reported results. ${ }^{1-5,9}$

From the movement of the dip position in the gain evolutions it is also found that, as the cavity length is shortened, the dye pulse arrives slightly early at the dye jet. It seems that 


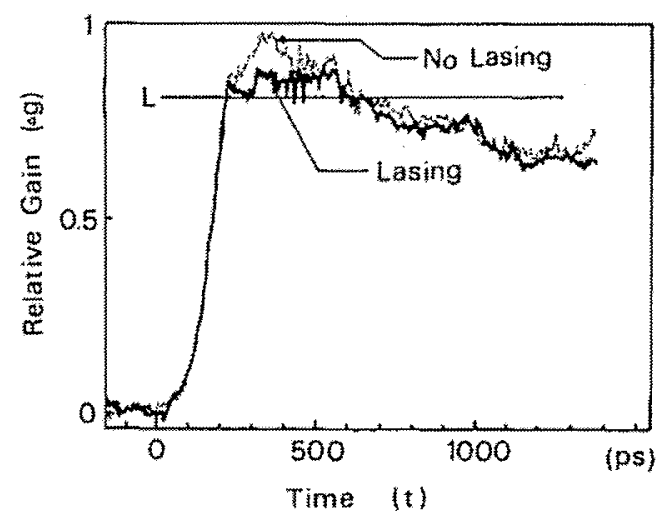

Fig. 3. Measured gain evolutions during SML action (solid line) and no laser action (dotted line) under the low pumping power. A narrow horizontal line $L$ represents the threshold level in SML action.

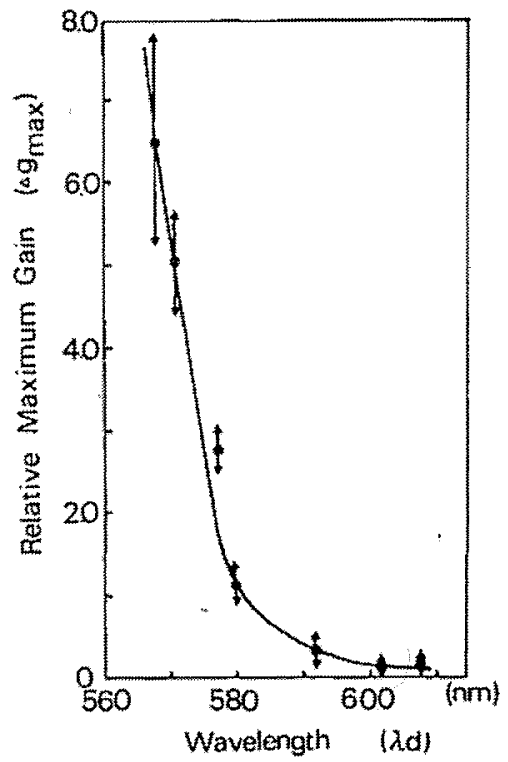

Fig. 4. Wavelength dependence of the gain peak for a rhodamine $6 \mathrm{G}$ jet stream in the case where laser action was stopped.

when the dye pulse arrives at the jet after the gain has risen above the maximum gain slope corresponding to the pump pulse peak and above the loss line but before its gain would reach the peak, the shortest duration pulse is produced. In this case the rising gain before the dye pulse arrives at the jet and the residual gain after it has done so show approximately the same height. This fact corresponds possibly to the fact that at the optimum cavity length the dye pulse suffers from equal net loss in both the leading and trailing edges of the pulse. As indicated already by theory, ${ }^{8-10,12-13}$ this is essential for mode locking.

We also carried out an additional experiment where another SML $\mathrm{cw}$ dye laser was constructed to elucidate the difference between the gain evolution during ML laser action and without laser action. The ML Ar-ion laser beam is split into two beams (1:1) by a beam splitter. One beam is used for the excitation source of the HML $\mathrm{cw}$ dye laser providing probe pulses, and the other after being chopped at $3.3 \mathrm{kHz}$ is used for the excitation source of another dye jet (gain sample of 1 $\times 10^{-3}$-M rhodamine $6 \mathrm{G}$ in ethylene glycol) placed in the SML laser cavity of the usual four-mirror-type arrangement. Output pulses from the HML dye laser after being optically delayed are combined with the chopped argon pulses which induce the gain to be measured by a dichroic mirror. The two collinear beams are focused through a lens onto the gain sample in the SML laser cavity. After passing through the gain sample and the glass filter, only the amplified probe beam is focused onto the photodiode detector. For measuring the induced gain as an ac component present in the chopping frequency of the probe beam, the output from the detector is connected to a lock-in amplifier (PAR 124A).

The measured gain evolution during SML action and that without laser action under the same low pumping power $(\sim 1.2$ times the threshold power) are shown by solid and dotted lines in Fig. 3, respectively. The narrow horizontal line $L$ represents the loss level of the SML laser which was estimated from the comparison between the pumping power at the operational condition and that at the threshold. From the dotted line it is found that when laser action is stopped the gain first rises with a rising time of $\sim 130 \mathrm{psec}$ and then drops slowly with $\sim 2.5$-nsec dye lifetime. ${ }^{14}$ This rising behavior corresponds to the fact that the gain around the arrival of the pump pulse under no laser action is proportional to the integral of the intensity of the pump pulse, which was suggested in Ref. 9 . The total evolution is similar to that obtained in a rhodamine $B$ solution except for the quantitative values. ${ }^{11}$ From the comparison between dotted and solid lines it is also found that the gain during mode-locking action is depleted in the vicinity of the peak due to stimulated emission by the intracavity dye pulse. However, in the present experimental conditions it is difficult to define distinctly the arrival time of the dye pulse in the gain evolution because of the relatively low pumping power.

Furthermore, the wavelength dependence of the gain peak in the case of no laser action was examined (see Fig. 4). For wavelengths shorter than $567 \mathrm{~nm}$, however, the gain measurement could not be made since the power of the probe pulse in its wavelength region was too low. Figure 4 shows that the gain increases rapidly in the wavelength region shorter than $580 \mathrm{~nm}$ in contrast to the wavelength dependence of the usual dye laser output whose blue shift was also observed for a rhodamine $B$ solution. ${ }^{11}$ This difference is presumably due to the following fact: in the case of a measured single-pass gain the reabsorption loss of the dye itself at the long-wavelength edge of the absorption spectra where the spectra overlap with the fluorescence spectra ${ }^{15}$ is much less effective than that due to the multiple-pass effect in the cavity in the case of laser action.

To summarize: it has been demonstrated that a continuous pump- and gain-probe technique is useful for an experimental study on a picosecond time scale of synchronous-passive hybrid mode-locking mechanisms in a $\mathrm{cw}$ dye laser. Cavity length detuning characteristics of the time evolution of the gain have been discussed in connection with the pulse autocorrelation trace. In addition, the difference between gain evolutions during mode locking and no laser action has been experimentally confirmed. The wavelength dependence of the small-signal gain has also been examined.

We gratefully acknowledge N. Haga and T. Katakura for their help with the experimental work. 


\section{References}

1. J. P. Heritage and R. K. Jain, Appl. Phys. Lett. 32, 101 (1978).

2. J.P. Ryan, L. S. Goldbery, and D. J. Bradley, Opt. Commun. 27, 127 (1978)

3. J. Kuhl, H. Klingenberg, and D. von der Linde, Appl. Phys. 18, 279 (1979).

4. G. W. Fehrenbach, K. J. Gruntz, and R. G. Ulbrich, Appl. Phys. Lett. 33, 159 (1978)

5. M. Yamashita, Rev. Laser Eng. 9, 210 (1981), in Japanese.

6. M. Yamashita, W. Sibbett, D. Welford, and D. J. Bradley, J. Appl. Phys. 51, 3559 (1980).

7. J. R. Taylor, M. C. Adams, and W. Sibbett, Appl. Phys. Lett. 35, 590 (1979).

8. N. J. Frigo, T. Daly, and H. Mahr, IEEE J. Quantum Electron. QE-13, 101 (1977).

9. C. P. Ausschnitt, R. K. Jain, and J. P. Heritage, IEEE J. Quantum Electron. QE-15, 912 (1979)

10. M. Yamashita, K. Yamada, and T. Sato, IEEE J. Quantum Electron. QE-18, 95 (1982).

11. W. T. Barnes and F. E. Lytle, Appl. Phys. Lett. 34, 509 (1979)

12. H. A. Haus, IEEE J. Quantum Electron. QE-11, 736 (1975).

13. H. A. Haus, IEEE J. Quantum Electron. QE-11, 323 (1975).

14. H. E. Lessing and A. V. Jena, "Continuous Picosecond Spectroscopy of Dyes," in Laser Handbook, Vol. 3, M. L. Stitch, Ed. (North-Holland, Amsterdam, 1979), pp. 753-846.

15. I. S. Berlman, Handbook of Fluorescence Spectra of Aromatic Molecules (Academic, New York, 1971), p. 412. 